\title{
METHOD OF SYNTHESIS NOVEL N'-SUBSTITUTED- 2-((5-(THIOPHEN-2-YLMETHYL)-4H-1,2,4-TRIAZOL-3-YL)THIO) ACETOHYDRAZIDES
}

\author{
YENİ N'-SÜBSTITÜE-2-((5-(TIYOFEN-2-ILMETIL)-4H-1,2,4-TRİAZOL-3-IL)TIYYO) \\ ASETOHIDRAZITLERIN SENTEZ YÖNTEMI
}

\begin{abstract}
Andrii A. SAFONOV*
Zaporizhzhia State Medical University, Faculty of Farmacy, Department of Natural Sciences for Foreign Students and Toxicological Chemistry, Ukraine
\end{abstract}

\begin{abstract}
Objective: The purpose of this work is to synthesize new series of $N^{\prime}$-substituted-2-((5-(thiophen-2ylmethyl)-4H-1,2,4-triazol-3-yl)thio)acetohydrazides.

Material and Method: The compound (3) (2-((3-(thiophen-2-ylmethyl)-1H-1,2,4-triazol-5yl)thio)acetohydrazide) was synthesized by adding hydrazine hydrate to isopropyl 2-((3-(thiophen-2-ylmethyl)$1 \mathrm{H}-1,2,4-$ triazol-5-yl)thio)acetate (1) and refluxed for 3-4 h in propan-2-ol. Synthesis of (5a-s) ( $N^{\prime}-$ substituted2-((3-(thiophen-2-ylmethyl)-1H-1,2,4-triazol-5-yl)thio)acetohydrazide) was carried out by adding aromatic or heterocyclic aldehyde to 2-((3-(thiophen-2-ylmethyl)-1H-1,2,4-triazol-5-yl)thio)acetohydrazide (3) in acetic acid. The structure of synthesized compounds is confirmed using Elemental analysis (CHNS), ${ }^{1} H-N M R$ and Chromatographic mass spectral analysis.

Result and Discussion: The compound (3) was synthesized by adding hydrazine hydrate to compound (1) and compound (3) characterized by ${ }^{1} H$-NMR peak, as a singlet due to (NH2) at $\delta=2.01$ ppm. Synthesis of (5a-s) was carried out by adding aromatic or heterocyclic aldehyde to compound (3) in acetic acid. The signals of $1 H$ NMR for (5a-s) are consented with the proposed structure. The elemental analysis (CHNS) was accomplished for synthesized compounds to confirm their basic chemical structures and revealed acceptable agreement with the calculated percentages.
\end{abstract}

Keywords: ${ }^{l} H$-NMR, heterocyclic compounds, synthesis, 1,2,4-triazole

ÖZ

Amaç: Bu çalışmanın amacı, bir seri yeni N'-sübstitüe-2-((5-(tiyofen-2-ilmetil)-4H-1,2,4-triazol-3il)tiyo)asetohidrazit sentezlemektir.

Gereç ve Yöntem: Bileşik (3) (2-((3-(tiyofen-2-ilmetil)-1H-1,2,4-triazol-5-il)tiyo)asetohidrazit), izopropil 2-((3-(tiyofen-2-ilmetil)-1H-1,2,4-triazol-5-il)tiyo)asetat (1)'e hidrazin hidrat ilave edilerek sentezlenmiş ve

\footnotetext{
* Corresponding Author/Sorumlu Yazar: Andrii A. Safonov e-mail/e-posta: 8safonov@gmail.com
} 
propan-2-ol içerisinde 3-4 saat geri çeviren soğutucuda bekletilmiştir. Bileşikler (5a-s) (N'-sübstitüe-2-(((3(tiyofen-2-ilmetil)-1H-1,2,4-triazol-5-il)tiyo)asetohidrazit), $\quad 3-(t i y o f e n-2-i l m e t i l)-1 H-1,2,4-t r i a z o l-5-$ il)tiyo)asetohidrazit (3) aromatik veya heterosiklik aldehit ilave edilerek sentezlenmiştir. Çözücü olarak asetik asit kullanılmıştır. Sentezlenen bileşiklerin yapısı, Elementel analiz (CHNS), ${ }^{l} H-N M R$ ve Kromatografik kütle spektral analizi kullanilarak doğrulanmıştır.

Sonuç ve Tartışma: Bileşik (3), Bileşik (1)'e hidrazin hidrat ilave edilerek sentezlenmiş ve $\mathrm{NH}=2,01$ ppm'de $\left(\mathrm{NH}_{2}\right)$ nedeniyle bir singlet formunda ${ }^{l} \mathrm{H}$-NMR pik ile karakterize edilmiştir. Bileşikler (5a-s), Bileşik (3)'e aromatik veya heterosiklik aldehit ilave edilerek asetik asit ortamda sentezlenmiştir. Bileşikler (5a-s) için önerilen yapı, ${ }^{1} \mathrm{H}$-NMR sinyalleri ile doğrulanmıştır. Temel kimyasal yapılarını doğrulamak için sentezlenen bileşikler üzerinde elementel analiz (CHNS) yapılmış ve hesaplanan yüzdelerle kabul edilebilir bir uyum sağladı̆̆ gösterilmiştir.

Anahtar Kelimeler: ${ }^{1} H-N M R$, heterosiklik bileşikler, sentez, 1,2,4-triazol

\section{INTRODUCTION}

The search for means to combat diseases was conducted throughout the history of human existence. However, if in the past medical preparations were made, as a rule, from substances of animal and vegetable origin, today the achievements in the field of synthetic organic chemistry are used for the production of medicines.

Modern science is linked to the creation of the necessary synthetic drugs [1, 2, 3]. As a result, it is possible to treat many diseases that were previously considered incurable or fatal $[4,5]$.

Synthetic drugs in modern medicine are an important, dominant group. Substances that receive synthetically contain less impurities than the extract from the plant material. Chemical preparations are subject to higher requirements than herbal preparations. A more rigorous assessment shall be made of their medicinal properties and the conditions of use.

Synthesis of 1,2,4-triazole derivatives is a promising direction for the creation of new substances $[6,7,8]$ with different types of biological activity $[9,10,11]$.

The aim of the work was to synthesize and to confirm structure of N'-substituted -2-((5-(thiophen2-ylmethyl)-4H-1,2,4-triazol-3-yl)thio)acetohydrazides.

\section{MATERIAL AND METHOD}

\section{Chemicals}

The initial compounds isopropyl 2-((3-(thiophen-2-ylmethyl)-1H-1,2,4-triazol-5-yl)thio)acetate and (1) were synthesized at the Department of Toxicological and Inorganic Chemistry of the Zaporizhzhya State Medical University (Ukraine) and purified by recrystallization with content of the main component $\geq 98 \%$ [12]. The hydrazine hydrate (assay- 50-60\%), acetic acid (99\%), benzaldehyde (99.5\%), 2-bromobenzaldehyde (98\%), 2-chlorobenzaldehyde (99\%), 4-chlorobenzaldehyde (97\%), 4fluorobenzaldehyde (98\%), 2-hydroxybenzaldehyde (98\%), 4-hydroxybenzaldehyde (98\%), 3methoxybenzaldehyde (98\%), 2-nitrobenzaldehyde (98\%), 3-nitrobenzaldehyde (98\%), 4- 
(dimethylamino)benzaldehyde (99\%), 2,4-dimethylbenzaldehyde (90\%), 2,3-dimethoxybenzaldehyde (98\%), 3,4-dimethoxybenzaldehyde (99\%), 3,5-dimethoxybenzaldehyde (98\%), 3-bromo-4fluorobenzaldehyde (98\%), nicotinaldehyde (98\%), thiophene-2-carbaldehyde (95\%), 5-nitrofuran-2carbaldehyde (90\%) and 2-propanol (99,5\%) were obtained from SIGMA-ALDRICH (Germany).

All chemicals and solvents used during synthesis were of analytical grade and used without further purification.

\section{Equipment}

The melting temperature was determined on an automatic melting device OptiMelt Stanford Research Systems MPA100 (US production). The elemental analysis of the compounds is installed on the elemental analyzer Elementar Vario L cube (CHNS) (standard - sulfanilamide) (Analysensysteme $\mathrm{GmbH}$, Germany). Chromatographic mass spectral studies were performed on Agilent 1260 Infinity HPLC liquid chromatograph equipped with an Agilent 6120 mass spectrometer (ionization in an electric spray (ESI) (US production), ${ }^{1} H$-NMR spectra were recorded on a Mercury 400 spectrometer (Umatek International Inc.), DMSO- $d_{6}$ solvent, internal standard - tetramethylsilane and deciphered using the ADVASP 143 computer program. The synthetic method is outlined in scheme $1[13,14,15]$.

\section{Chemical Synthesis}

General method for synthesis of 2-((3-(thiophen-2-ylmethyl)-1H-1,2,4-triazol-5yl)thio)acetohydrazide (3)

A mixture of isopropyl 2-((3-(thiophen-2-ylmethyl)-1H-1,2,4-triazol-5-yl)thio)acetate (1) (0.1 $\mathrm{mol}$ ) and hydrazine hydrate $(0.15 \mathrm{~mol})$ (2) were dissolved in $250 \mathrm{ml}$ propan-2-ol in a round bottom flask and refluxed for 3-4 h. After completion of the reaction, the mixture was evaporated. The residue obtained was dried, recrystallized from propan-2-ol.

\section{2-((3-(Thiophen-2-ylmethyl)-1H-1,2,4-triazol-5-yl)thio)acetohydrazide (3)}

Bright yellow powder; yield 72\%; m.p. 124-1260C ; 1HNMR (400 MHz, DMSO-d6, $\delta=$ ppm): $13.51(1 \mathrm{H}, \mathrm{s}, \mathrm{NH}) ; 8,02(1 \mathrm{H}, \mathrm{s}, \mathrm{NH}) ; 7.31(1 \mathrm{H}, \mathrm{d}$, thiophen-H); $6.87(1 \mathrm{H}, \mathrm{t}$, thiophen-H); $6.72(1 \mathrm{H}, \mathrm{d}$, thiophen-H); 3.69 (2H, s, CH2); 3.50 (2H, s, CH2); 2.01 (2H, s, NH2); CHNS elemental analysis Calcd. for (C9H11N5OS2): found C\% 40.10, H\% 4.10, N\% 26.05, S\% 23.79; calculated C\% 40.13, H\% 4.12, N\% 26.00, S\% 23.81. Peak of a pseudo-molecular ion $[\mathrm{MH}]+270$.

Synthesis of titled compounds (5a-5s)

A mixture of 2-((3-(thiophen-2-ylmethyl)-1H-1,2,4-triazol-5-yl)thio)acetohydrazide (3) (0.01 mol, $2.69 \mathrm{~g}$ ) and aromatic or heterocyclic aldehyde (4) (0.01 mol): benzaldehyde (1.06g) or 2bromobenzaldehyde $(1.85 \mathrm{~g})$ or 2-chlorobenzaldehyde $(1.40 \mathrm{~g})$ or 4 -chlorobenzaldehyde $(1.40 \mathrm{~g})$ or 4 fluorobenzaldehyde (1.24g) or 2-hydroxybenzaldehyde (1.22g) or 4-hydroxybenzaldehyde (1.22g) or 3- 
methoxybenzaldehyde $(1.36 \mathrm{~g})$ or 2-nitrobenzaldehyde $(1.51 \mathrm{~g})$ or 3-nitrobenzaldehyde $(1.51 \mathrm{~g})$ or 4(dimethylamino)benzaldehyde $\quad(1.49 \mathrm{~g})$ or 2,4 -dimethylbenzaldehyde $\quad(1.34 \mathrm{~g})$ or 2,3 dimethoxybenzaldehyde (1.66g) or 3,4-dimethoxybenzaldehyde (1.66g) or 3,5-dimethoxybenzaldehyde (1.66g) or 3-bromo-4-fluorobenzaldehyde $(2.01 \mathrm{~g})$ or nicotinaldehyde $(1.07 \mathrm{~g})$ or thiophene-2carbaldehyde $(1.12 \mathrm{~g})$ or 5-nitrofuran-2-carbaldehyde (1.41g) in concentrated acetic acid was heated until the solid was dissolved. After cooling the residue was formed and washed by diethyl ether, filtered and dried, then recrystallized from acetic acid.

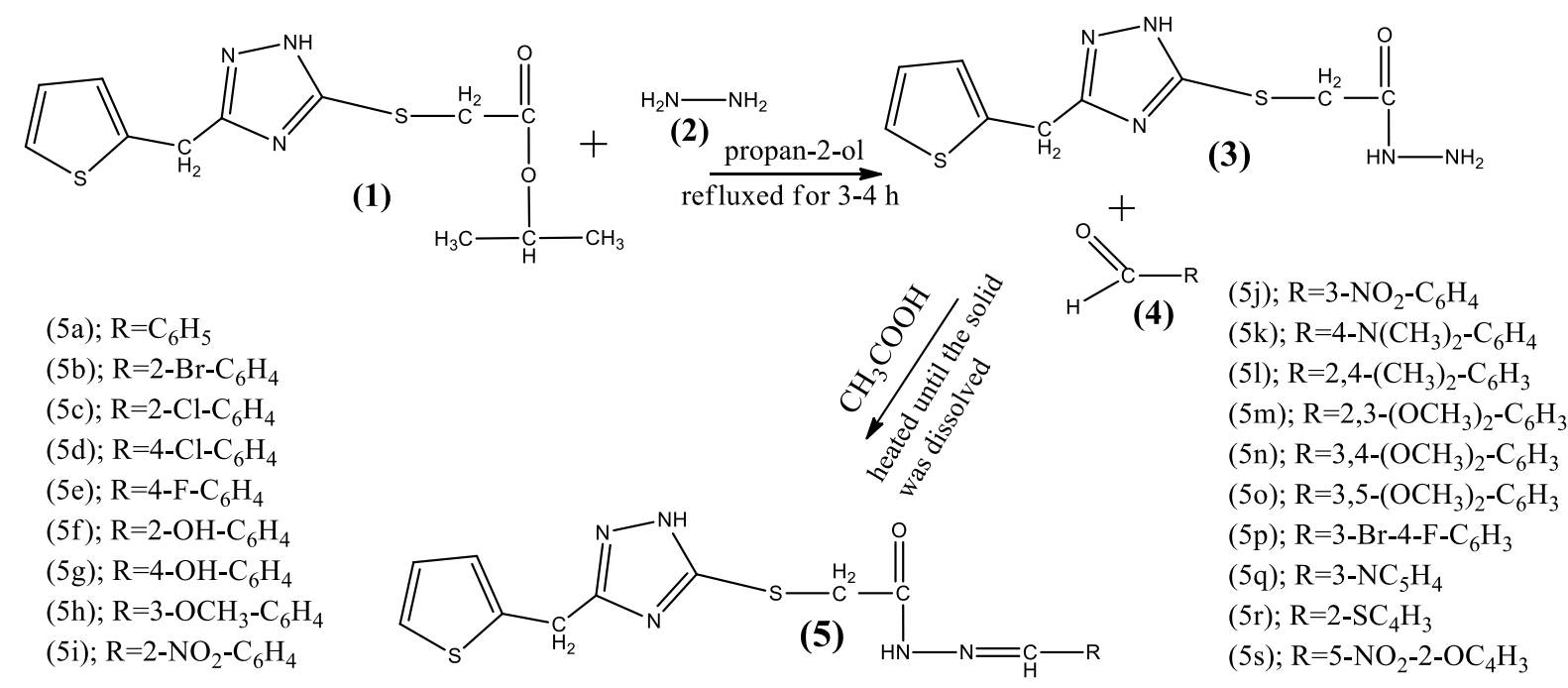

Scheme 1: Synthesis of 1,2,4-triazole derivatives 3-5s

N'-Benzylidene-2-((3-(thiophen-2-ylmethyl)-1H-1,2,4-triazol-5-yl)thio)acetohydrazide (5a)

Yellow powder; yield 70\%; m.p. 56-580C ; 1HNMR (400 MHz, DMSO-d6, $\delta=$ ppm): $13.52(1 \mathrm{H}$, s, NH); $8.81(1 \mathrm{H}, \mathrm{s}, \mathrm{CH}), 8,04(1 \mathrm{H}, \mathrm{s}, \mathrm{NH}) ; 7.81(2 \mathrm{H}, \mathrm{d}, \operatorname{Ar}-\mathrm{H}), 7,51(3 \mathrm{H}, \mathrm{m}, \operatorname{Ar}-\mathrm{H}), 7.30(1 \mathrm{H}, \mathrm{d}$, thiophen-H); $6.86(1 \mathrm{H}, \mathrm{t}$, thiophen-H); $6.71(1 \mathrm{H}, \mathrm{d}$, thiophen-H); $3.78(2 \mathrm{H}, \mathrm{s}, \mathrm{CH} 2) ; 3.59(2 \mathrm{H}, \mathrm{s}, \mathrm{CH} 2)$; CHNS elemental analysis Calcd. for (C16H15N5OS2) : found C\% 53.70, H\% 4.21, N\% 19.56, S\% 17.96; calculated C\% 53.76, H\% 4.23, N\% 19.59, S\% 17.94. Peak of a pseudo-molecular ion [MH]+ 358.

N'-(2-Bromobenzylidene)-2-((3-(thiophen-2-ylmethyl)-1H-1,2,4-triazol-5-yl)thio)acetohydrazide $(5 b)$

Orange powder; yield 79\%; m.p. 114-1160C ; 1HNMR (400 MHz, DMSO-d6, $\delta=$ ppm): 13.50 $(1 \mathrm{H}, \mathrm{s}, \mathrm{NH}) ; 8.75(1 \mathrm{H}, \mathrm{s}, \mathrm{CH}), 8,06(1 \mathrm{H}, \mathrm{s}, \mathrm{NH}) ; 7.81(1 \mathrm{H}, \mathrm{d}, \mathrm{Ar}-\mathrm{H}), 7,57(1 \mathrm{H}, \mathrm{d}, \mathrm{Ar}-\mathrm{H}), 7.34(\mathrm{~m}, 1 \mathrm{H}$, thiophen-H, 2H, Ar-H); $6.85(1 \mathrm{H}, \mathrm{t}$, thiophen-H); $6.71(1 \mathrm{H}, \mathrm{d}$, thiophen-H); $3.79(2 \mathrm{H}, \mathrm{s}, \mathrm{CH} 2) ; 3.61$ (2H, s, CH2); CHNS elemental analysis Calcd. for (C16H14BrN5OS2) : found C\% 44.12, H\% 3.25, N\% 16.09, S\% 14.72; calculated C\% 44.04, H\% 3.23, N\% 16.05, S\% 14.70. Peak of a pseudo-molecular ion $[\mathrm{MH}]+437$. 
N'-(2-Chlorobenzylidene)-2-((3-(thiophen-2-ylmethyl)-1H-1,2,4-triazol-5-

yl)thio)acetohydrazide (5c)

Yellow powder; yield 74\%; m.p. 161-1630C ; 1HNMR (400 MHz, DMSO-d6, $\delta=p p m): ~ 13.51$ $(1 \mathrm{H}, \mathrm{s}, \mathrm{NH}) ; 8.72(1 \mathrm{H}, \mathrm{s}, \mathrm{CH}), 8,02(1 \mathrm{H}, \mathrm{s}, \mathrm{NH}) ; 7.80(1 \mathrm{H}, \mathrm{d}, \mathrm{Ar}-\mathrm{H}), 7,51(1 \mathrm{H}, \mathrm{d}, \mathrm{Ar}-\mathrm{H}), 7.30(\mathrm{~m}, 1 \mathrm{H}$, thiophen-H, 2H, Ar-H); 6.89 (1H, t, thiophen-H); $6.71(1 \mathrm{H}, \mathrm{d}$, thiophen-H); $3.85(2 \mathrm{H}, \mathrm{s}, \mathrm{CH} 2) ; 3.58$ (2H, s, CH2); CHNS elemental analysis Calcd. for (C16H14ClN5OS2) : found C\% 49.12, H\% 3.63, N\% 17.91, S\% 16.39; calculated C\% 49.04, H\% 3.60, N\% 17.87, S\% 16.36. Peak of a pseudo-molecular ion $[\mathrm{MH}]+393$.

$N^{\prime}$-(4-Chlorobenzylidene)-2-((3-(thiophen-2-ylmethyl)-1H-1,2,4-triazol-5-

yl)thio)acetohydrazide (5d)

Bright yellow powder; yield 79\%; m.p. 189-1910C ; 1HNMR (400 MHz, DMSO-d6, $\delta=$ ppm): $13.56(1 \mathrm{H}, \mathrm{s}, \mathrm{NH}) ; 8.81(1 \mathrm{H}, \mathrm{s}, \mathrm{CH}), 7,99(1 \mathrm{H}, \mathrm{s}, \mathrm{NH}) ; 7.80(2 \mathrm{H}, \mathrm{d}, \mathrm{Ar}-\mathrm{H}), 7,52$ (2H, d, Ar-H), 7.38 (d, $1 \mathrm{H}$, thiophen- $\mathrm{H}) ; 6.86(1 \mathrm{H}, \mathrm{t}$, thiophen- $\mathrm{H}) ; 6.71(1 \mathrm{H}, \mathrm{d}$, thiophen- $\mathrm{H}) ; 3.65(2 \mathrm{H}, \mathrm{s}, \mathrm{CH} 2) ; 3.52(2 \mathrm{H}, \mathrm{s}$, $\mathrm{CH} 2)$; CHNS elemental analysis Calcd. for (C16H14CIN5OS2) : found C\% 49.12, H\% 3.62, N\% 17.89, S\% 16.38; calculated C\% 49.04, H\% 3.60, N\% 17.87, S\% 16.36. Peak of a pseudo-molecular ion $[\mathrm{MH}]+$ 393.

N'-(4-Fluorobenzylidene)-2-((3-(thiophen-2-ylmethyl)-1H-1,2,4-triazol-5-yl)thio)acetohydrazide $(5 e)$

Yellow powder; yield 76\%; m.p. 166-1680C ; 1HNMR (400 MHz, DMSO-d6, $\delta=p p m): ~ 13.48$ $(1 \mathrm{H}, \mathrm{s}, \mathrm{NH}) ; 8.88(1 \mathrm{H}, \mathrm{s}, \mathrm{CH}), 8,00(1 \mathrm{H}, \mathrm{s}, \mathrm{NH}) ; 7.76(2 \mathrm{H}, \mathrm{d}, \mathrm{Ar}-\mathrm{H}), 7,48(2 \mathrm{H}, \mathrm{d}, \mathrm{Ar}-\mathrm{H}), 7.32(\mathrm{~d}, 1 \mathrm{H}$, thiophen-H); $6.80(1 \mathrm{H}, \mathrm{t}$, thiophen-H); $6.62(1 \mathrm{H}, \mathrm{d}$, thiophen-H); $3.71(2 \mathrm{H}, \mathrm{s}, \mathrm{CH} 2) ; 3.52(2 \mathrm{H}, \mathrm{s}, \mathrm{CH} 2)$; CHNS elemental analysis Calcd. for (C16H14FN5OS2): found C\% 51.25, H\% 3.78, N\% 18.68, S\% 17.12; calculated C\% 51.19, H\% 3.76, N\% 18.65, S\% 17.08. Peak of a pseudo-molecular ion [MH]+ 376.

$N^{\prime}-(2-H y d r o x o b e n z y l i d e n e)-2-((3-(t h i o p h e n-2-y l m e t h y l)-1 H-1,2,4-t r i a z o l-5-$

yl)thio)acetohydrazide (5f)

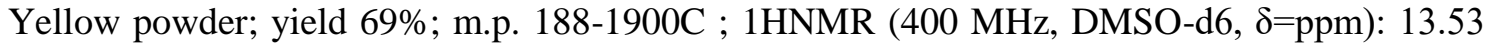
$(1 \mathrm{H}, \mathrm{s}, \mathrm{NH}) ; 8.78(1 \mathrm{H}, \mathrm{s}, \mathrm{CH}), 7,96(1 \mathrm{H}, \mathrm{s}, \mathrm{NH}) ; 7.68(1 \mathrm{H}, \mathrm{d}, \mathrm{Ar}-\mathrm{H}), 7,51(1 \mathrm{H}, \mathrm{t}, \mathrm{Ar}-\mathrm{H}), 7.34(\mathrm{~d}, 1 \mathrm{H}$, thiophen-H); 7,11 (2H, m, Ar-H), $6.89(1 \mathrm{H}, \mathrm{t}$, thiophen- $\mathrm{H}) ; 6.71(1 \mathrm{H}, \mathrm{d}$, thiophen- $\mathrm{H}) ; 5,41(1 \mathrm{H}, \mathrm{s}, \mathrm{OH})$; $3.95(2 \mathrm{H}, \mathrm{s}, \mathrm{CH} 2) ; 3.67$ (2H, s, CH2); CHNS elemental analysis Calcd. for (C16H15N5O2S2) : found C\% 51.37, H\% 4.03, N\% 18.69, S\% 17.13; calculated C\% 51.46, H\% 4.05, N\% 18.75, S\% 17.17. Peak of a pseudo-molecular ion $[\mathrm{MH}]+374$.

$N^{\prime}$-(4-Hydroxobenzylidene)-2-((3-(thiophen-2-ylmethyl)-1H-1,2,4-triazol-5yl)thio)acetohydrazide (5g) 
Orange powder; yield 73\%; m.p. 180-1820C ; 1HNMR (400 MHz, DMSO-d6, $\delta=p p m): ~ 13.54$ $(1 \mathrm{H}, \mathrm{s}, \mathrm{NH}) ; 8.38(1 \mathrm{H}, \mathrm{s}, \mathrm{CH}) ; 8,03(1 \mathrm{H}, \mathrm{s}, \mathrm{NH}) ; 7.74(2 \mathrm{H}, \mathrm{d}, \mathrm{Ar}-\mathrm{H}), 7.34(\mathrm{~d}, 1 \mathrm{H}$, thiophen-H); 6.89 $(1 \mathrm{H}, \mathrm{t}$, thiophen-H); $6.71(1 \mathrm{H}, \mathrm{d}$, thiophen-H); $6.59(2 \mathrm{H}, \mathrm{m}, \mathrm{Ar}-\mathrm{H}), 5,34(1 \mathrm{H}, \mathrm{s}, \mathrm{OH}) ; 3.86(2 \mathrm{H}, \mathrm{s}, \mathrm{CH} 2)$; 3.59 (2H, s, CH2); CHNS elemental analysis Calcd. for (C16H15N5O2S2) : found C\% 51.52, $\mathrm{H} \% 4.08$, N\% 18.81, S\% 17.19; calculated C\% 51.46, H\% 4.05, N\% 18.75, S\% 17.17. Peak of a pseudo-molecular ion $[\mathrm{MH}]+374$.

$N^{\prime}$-(3-Methoxybenzylidene)-2-((3-(thiophen-2-ylmethyl)-1H-1,2,4-triazol-5yl)thio)acetohydrazide (5h)

Yellow powder; yield 76\%; m.p. 85-870C ; 1HNMR (400 MHz, DMSO-d6, $\delta=$ ppm): $13.51(1 \mathrm{H}$, s, NH); $8.54(1 \mathrm{H}, \mathrm{s}, \mathrm{CH}) ; 8,05(1 \mathrm{H}, \mathrm{s}, \mathrm{NH}) ; 7.57(1 \mathrm{H}, \mathrm{s}, \mathrm{Ar}-\mathrm{H}), 7.38(\mathrm{~m}, 1 \mathrm{H}$, thiophen-H, 2H, Ar-H); $7.06(1 \mathrm{H}, \mathrm{d}, \mathrm{Ar}-\mathrm{H}), 6.89(1 \mathrm{H}, \mathrm{t}$, thiophen-H); $6.71(1 \mathrm{H}, \mathrm{d}$, thiophen- $\mathrm{H}) ; 6.59(2 \mathrm{H}, \mathrm{m}, \mathrm{Ar}-\mathrm{H}), 5,34(1 \mathrm{H}$, s, OH); $3.86(2 \mathrm{H}, \mathrm{s}, \mathrm{CH} 2) ; 3.64(3 \mathrm{H}, \mathrm{s}, \mathrm{CH} 3) 3.59$ (2H, s, CH2); CHNS elemental analysis Calcd. for (C17H17N5O2S2) : found C\% 52.72, H\% 4.45, N\% 18.09, S\% 16.57; calculated C\% 52.69, H\% 4.42, $\mathrm{N} \% 18.07, \mathrm{~S} \%$ 16.55. Peak of a pseudo-molecular ion $[\mathrm{MH}]+388$.

$N^{\prime}$-(2-Nitrobenzylidene)-2-((3-(thiophen-2-ylmethyl)-1H-1,2,4-triazol-5-yl)thio)acetohydrazide

Yellow powder; yield 72\%; m.p. 124-1260C ; 1HNMR (400 MHz, DMSO-d6, $\delta=p p m): ~ 13.50$ $(1 \mathrm{H}, \mathrm{s}, \mathrm{NH}) ; 8.57(1 \mathrm{H}, \mathrm{s}, \mathrm{CH}), 8,09(1 \mathrm{H}, \mathrm{s}, \mathrm{NH}) ; 7.94(3 \mathrm{H}, \mathrm{m}, \mathrm{Ar}-\mathrm{H}), 7,52(1 \mathrm{H}, \mathrm{t}, \mathrm{Ar}-\mathrm{H}), 7.35(\mathrm{~d}, 1 \mathrm{H}$, thiophen-H); $6.87(1 \mathrm{H}, \mathrm{t}$, thiophen-H); $6.75(1 \mathrm{H}, \mathrm{d}$, thiophen- $\mathrm{H}) ; 3.84(2 \mathrm{H}, \mathrm{s}, \mathrm{CH} 2) ; 3.53(2 \mathrm{H}, \mathrm{s}, \mathrm{CH} 2)$; CHNS elemental analysis Calcd. for (C16H14N6O3S2) : found C\% 47.99, H\% 3.52, N\% 20.84, S\% 15.98; calculated C\% 47.79, H\% 3.53, N\% 20.90, S\% 15.91. Peak of a pseudo-molecular ion $[\mathrm{MH}]+$ 403.

N'-(3-Nitrobenzylidene)-2-((3-(thiophen-2-ylmethyl)-1H-1,2,4-triazol-5-yl)thio)acetohydrazide

Orange powder; yield 70\%; m.p. 98-1000C ; 1HNMR (400 MHz, DMSO-d6, $\delta=$ ppm): $13.52(1 \mathrm{H}$, s, NH); $8.61(1 \mathrm{H}, \mathrm{d}, \mathrm{Ar}-\mathrm{H}), 8.49(1 \mathrm{H}, \mathrm{s}, \mathrm{CH}), 8.14(2 \mathrm{H}, \mathrm{m}, \mathrm{Ar}-\mathrm{H}), 8,00(1 \mathrm{H}, \mathrm{s}, \mathrm{NH}) ; 7,74(1 \mathrm{H}, \mathrm{t}, \mathrm{Ar}-\mathrm{H})$, $7.34(\mathrm{~d}, 1 \mathrm{H}$, thiophen-H); $6.87(1 \mathrm{H}, \mathrm{t}$, thiophen- $\mathrm{H}) ; 6.72(1 \mathrm{H}, \mathrm{d}$, thiophen- $\mathrm{H}) ; 3.88(2 \mathrm{H}, \mathrm{s}, \mathrm{CH} 2) ; 3.51$ (2H, s, CH2); CHNS elemental analysis Calcd. for (C16H14N6O3S2) : found C\% 47.70, H\% 3.53, N\% 20.91, S\% 15.96; calculated C\% 47.75, H\% 3.51, N\% 20.88, S\% 15.93. Peak of a pseudo-molecular ion $[\mathrm{MH}]+403$.

N'-(4-Dimethylaminobenzylidene)-2-((3-(thiophen-2-ylmethyl)-1H-1,2,4-triazol-5yl)thio)acetohydrazide ( $5 k)$

Orange powder; yield 78\%; m.p. 121-1230C ; 1HNMR (400 MHz, DMSO-d6, $\delta=$ ppm): 13.54 $(1 \mathrm{H}, \mathrm{s}, \mathrm{NH}) ; 8.55(1 \mathrm{H}, \mathrm{s}, \mathrm{CH}), 7,92(1 \mathrm{H}, \mathrm{s}, \mathrm{NH}) ; 7.45(2 \mathrm{H}, \mathrm{d}, \mathrm{Ar}-\mathrm{H}), 7.38(\mathrm{~d}, 1 \mathrm{H}$, thiophen-H); 6.89 (1H, t, thiophen-H); 6,81 (2H, d, Ar-H), $6.72(1 \mathrm{H}, \mathrm{d}$, thiophen-H); $3.65(2 \mathrm{H}, \mathrm{s}, \mathrm{CH} 2) ; 3.48(2 \mathrm{H}, \mathrm{s}, \mathrm{CH} 2)$; 
3.02 (6H, s, 2CH3); CHNS elemental analysis Calcd. for (C18H20N6OS2) : found C\% 53.91, H\% 5.05, N\% 20.99, S\% 16.03; calculated C\% 53.98, H\% 5.03, N\% 20.98, S\% 16.01. Peak of a pseudo-molecular ion $[\mathrm{MH}]+401$.

$N^{\prime}$-(2,4-Dimethylbenzylidene)-2-((3-(thiophen-2-ylmethyl)-1H-1,2,4-triazol-5-

yl)thio)acetohydrazide (5l)

Yellow powder; yield 68\%; m.p. 106-1080C ; 1HNMR (400 MHz, DMSO-d6, $\delta=$ ppm): 13.45 $(1 \mathrm{H}, \mathrm{s}, \mathrm{NH}) ; 8.88(1 \mathrm{H}, \mathrm{s}, \mathrm{CH}), 8,00(1 \mathrm{H}, \mathrm{s}, \mathrm{NH}) ; 7.78(1 \mathrm{H}, \mathrm{d}, \mathrm{Ar}-\mathrm{H}) ; 7.38(\mathrm{~d}, 1 \mathrm{H}$, thiophen-H); 7.05 $(2 \mathrm{H}, \mathrm{s}, \mathrm{Ar}-\mathrm{H}), 6.89(1 \mathrm{H}, \mathrm{t}$, thiophen-H); $6.75(1 \mathrm{H}, \mathrm{d}$, thiophen- $\mathrm{H}) ; 3.70(2 \mathrm{H}, \mathrm{s}, \mathrm{CH} 2) ; 3.50(2 \mathrm{H}, \mathrm{s}, \mathrm{CH} 2)$; 2.41 (3H, s, CH3); 2.24 (3H, s, CH3); CHNS elemental analysis Calcd. for (C18H19N5OS2) : found C\% 56.12, H\% 4.99, N\% 18.20, S\% 16.61; calculated C\% 56.08, H\% 4.97, N\% 18.17, S\% 16.64. Peak of a pseudo-molecular ion $[\mathrm{MH}]+386$.

N'-(2,3-Dimethoxybenzylidene)-2-((3-(thiophen-2-ylmethyl)-1H-1,2,4-triazol-5yl)thio)acetohydrazide (5m)

Yellow powder; yield 75\%; m.p. 131-1320C ; 1HNMR (400 MHz, DMSO-d6, $\delta=p p m): ~ 13.51$ $(1 \mathrm{H}, \mathrm{s}, \mathrm{NH}) ; 8.80(1 \mathrm{H}, \mathrm{s}, \mathrm{CH}), 8,10(1 \mathrm{H}, \mathrm{s}, \mathrm{NH}) ; 7.40(1 \mathrm{H}, \mathrm{d}$, thiophen-H); $7.26(1 \mathrm{H}, \mathrm{d}, \mathrm{Ar}-\mathrm{H}) ; 7.08$ $(1 \mathrm{H}, \mathrm{d}, \mathrm{Ar}-\mathrm{H}) ; 6.87(\mathrm{~m}, 1 \mathrm{H}$, thiophen-H, 1H, Ar-H); $6.71(1 \mathrm{H}, \mathrm{d}$, thiophen-H); $3.74(2 \mathrm{H}, \mathrm{s}, \mathrm{CH} 2) ; 3.52$ (2H, s, CH2); 3.44 (6H, s, CH3); CHNS elemental analysis Calcd. for (C18H19N5O3S2) : found C\% 51.84, H\% 4.57, N\% 16.79, S\% 15.40; calculated C\% 51.78, H\% 4.59, N\% 16.77, S\% 15.36. Peak of a pseudo-molecular ion $[\mathrm{MH}]+418$.

N'-(3,4-Dimethoxybenzylidene)-2-((3-(thiophen-2-ylmethyl)-1H-1,2,4-triazol-5-

$y l$ )thio)acetohydrazide $(5 n)$

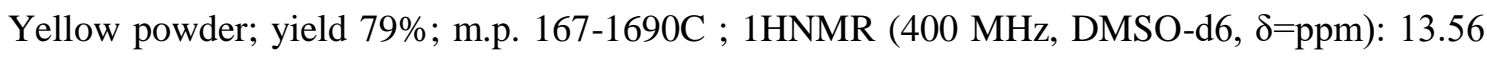
$(1 \mathrm{H}, \mathrm{s}, \mathrm{NH}) ; 8.72(1 \mathrm{H}, \mathrm{s}, \mathrm{CH}) ; 8,02(1 \mathrm{H}, \mathrm{s}, \mathrm{NH}) ; 7.59(1 \mathrm{H}, \mathrm{s}, \mathrm{Ar}-\mathrm{H}) ; 7.40(1 \mathrm{H}, \mathrm{d}$, thiophen-H); $7.28(1 \mathrm{H}$, d, Ar-H); 6.87 (m, 1H, thiophen-H, 1H, Ar-H); $6.72(1 \mathrm{H}, \mathrm{d}$, thiophen-H); $3.79(2 \mathrm{H}, \mathrm{s}, \mathrm{CH} 2) ; 3.54(2 \mathrm{H}$, s, CH2); 3.49 (6H, s, CH3); CHNS elemental analysis Calcd. for (C18H19N5O3S2) : found C\% 51.87, H\% 4.62, N\% 16.81, S\% 15.38; calculated C\% 51.78, H\% 4.59, N\% 16.77, S\% 15.36. Peak of a pseudomolecular ion $[\mathrm{MH}]+418$.

N'-(3,5-Dimethoxybenzylidene)-2-((3-(thiophen-2-ylmethyl)-1H-1,2,4-triazol-5-

yl)thio)acetohydrazide (5o)

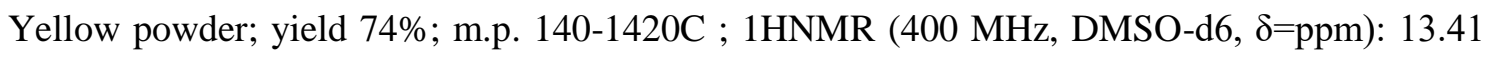
$(1 \mathrm{H}, \mathrm{s}, \mathrm{NH}) ; 8.64(1 \mathrm{H}, \mathrm{s}, \mathrm{CH}) ; 8,05(1 \mathrm{H}, \mathrm{s}, \mathrm{NH}) ; 7.40(1 \mathrm{H}, \mathrm{d}$, thiophen-H); $7.09(2 \mathrm{H}, \mathrm{s}, \mathrm{Ar}-\mathrm{H}) ; 6.87(1 \mathrm{H}$, t, thiophen-H); 6.72 (1H, d, thiophen-H); 6,53(s, 1H, Ar-H); 3.78 (2H, s, CH2); 3.54 (2H, s, CH2); 3.41 (6H, s, CH3); CHNS elemental analysis Calcd. for (C18H19N5O3S2) : found C\% 51.74, H\% 4.56, N\% 16.76, S\% 15.40; calculated C\% 51.78, H\% 4.59, N\% 16.77, S\% 15.36. Peak of a pseudo-molecular ion $[\mathrm{MH}]+418$. 
N'-(3-Bromo-4-fluorobenzylidene)-2-((3-(thiophen-2-ylmethyl)-1H-1,2,4-triazol-5yl)thio)acetohydrazide (5p)

Bright orange powder; yield 68\%; m.p. 148-1500C; 1HNMR (400 MHz, DMSO-d6, $\delta=$ ppm): $13.48(1 \mathrm{H}, \mathrm{s}, \mathrm{NH}) ; 8.39(1 \mathrm{H}, \mathrm{s}, \mathrm{CH}) ; 8,12(1 \mathrm{H}, \mathrm{s}, \mathrm{NH}) ; 7.94(1 \mathrm{H}, \mathrm{d}, \mathrm{Ar}-\mathrm{H}) ; 7.74(1 \mathrm{H}, \mathrm{m}, \mathrm{Ar}-\mathrm{H}) ; 7.40$ $(1 \mathrm{H}, \mathrm{d}$, thiophen-H); $7.21(1 \mathrm{H}, \mathrm{t}, \mathrm{Ar}-\mathrm{H}) ; 6.83(1 \mathrm{H}, \mathrm{t}$, thiophen- $\mathrm{H}) ; 6.70(1 \mathrm{H}, \mathrm{d}$, thiophen- $\mathrm{H}) ; 3.84(2 \mathrm{H}$, s, CH2); 3.69 (2H, s, CH2); CHNS elemental analysis Calcd. for (C16H13BrFN5OS2) : found C\% 42.36, H\% 2.86, N\% 15.44, S\% 14.09; calculated C\% 42.30, H\% 2.88, N\% 15.41, S\% 14.11. Peak of a pseudo-molecular ion $[\mathrm{MH}]+455$.

$N^{\prime}$-(Pyridin-3-ylmethylene)-2-((3-(thiophen-2-ylmethyl)-1H-1,2,4-triazol-5-

yl)thio)acetohydrazide (5q)

Yellow powder; yield 71\%; m.p. 101-1030C; 1HNMR (400 MHz, DMSO-d6, $\delta=$ ppm): 13.61 (1H, s, NH); 9.09 (1H, s, Pyr-H); 8.74 (1H, d, Pyr-H); 8.53 (1H, s, CH); 8.30 (1H, d, Pyr-H); 8,01 (1H, s, NH); $7.54(1 \mathrm{H}, \mathrm{t}$, Pyr-H); $7.32(1 \mathrm{H}, \mathrm{d}$, thiophen-H); $6.87(1 \mathrm{H}, \mathrm{t}$, thiophen-H); $6.72(1 \mathrm{H}, \mathrm{d}$, thiophen$\mathrm{H}) ; 3.98$ (2H, s, CH2); 3.69 (2H, s, CH2); CHNS elemental analysis Calcd. for (C15H14N6OS2) : found $\mathrm{C} \%$ 50.29, H\% 3.91, N\% 23.48, S\% 17.92; calculated C\% 50.26, H\% 3.94, N\% 23.43, S\% 17.89. Peak of a pseudo-molecular ion $[\mathrm{MH}]+359$.

2-((3-(Thiophen-2-ylmethyl)-1H-1,2,4-triazol-5-yl)thio)-N'-(thiophen-2-

ylmethylene)acetohydrazide (5r)

Bright brown powder; yield 67\%; m.p. 111-1130C; 1HNMR (400 MHz, DMSO-d6, $\delta=$ ppm): $13.50(1 \mathrm{H}, \mathrm{s}, \mathrm{NH}) ; 8.24(1 \mathrm{H}, \mathrm{s}, \mathrm{CH}) ; 8,09(1 \mathrm{H}, \mathrm{s}, \mathrm{NH}) ; 7.84(1 \mathrm{H}$, d, thiophen-H); $7.59(1 \mathrm{H}, \mathrm{d}$, thiophen$\mathrm{H}) ; 7.34(1 \mathrm{H}, \mathrm{d}$, thiophen-H); $7.11(1 \mathrm{H}, \mathrm{t}$, thiophen-H); $6.82(1 \mathrm{H}$, t, thiophen- $\mathrm{H}) ; 6.70(1 \mathrm{H}, \mathrm{d}$, thiophen$\mathrm{H}) ; 3.71$ (2H, s, CH2); 3.53 (2H, s, CH2); CHNS elemental analysis Calcd. for (C14H13N5OS3) : found C\% 46.31, H\% 3.61, N\% 19.29, S\% 26.49; calculated C\% 46.26, H\% 3.60, N\% 19.27, S\% 26.46. Peak of a pseudo-molecular ion $[\mathrm{MH}]+364$.

$N^{\prime}-((5-N i t r o f u r a n-2-y l) m e t h y l e n e)-2-((3-(t h i o p h e n-2-y l m e t h y l)-1 H-1,2,4-t r i a z o l-5-$

yl)thio)acetohydrazide $(5 s)$

Brown powder; yield $74 \%$; m.p. 158-1600C; 1HNMR (400 MHz, DMSO-d6, $\delta=$ ppm): 13.55 $(1 \mathrm{H}, \mathrm{s}, \mathrm{NH}) ; 8.44(1 \mathrm{H}, \mathrm{s}, \mathrm{CH}) ; 7,92(1 \mathrm{H}, \mathrm{s}, \mathrm{NH}) ; 7.59(1 \mathrm{H}, \mathrm{d}$, Fur-H); $7.30(1 \mathrm{H}, \mathrm{d}$, thiophen-H); 7.08 $(1 \mathrm{H}, \mathrm{d}$, Fur-H); $6.80(1 \mathrm{H}, \mathrm{t}$, thiophen-H); $6.68(1 \mathrm{H}, \mathrm{d}$, thiophen-H); $3.84(2 \mathrm{H}, \mathrm{s}, \mathrm{CH}) ; 3.62(2 \mathrm{H}, \mathrm{s}$, $\mathrm{CH} 2)$; CHNS elemental analysis Calcd. for (C14H12N6O4S2): found C\% 42.92, H\% 3.07, N\% 21.47, S\% 16.36; calculated C\% 42.85, H\% 3.08, N\% 21.44, S\% 16.39. Peak of a pseudo-molecular ion [MH]+ 394. 


\section{RESULT AND DISCUSSION}

The compound (3) (2-((3-(thiophen-2-ylmethyl)-1H-1,2,4-triazol-5-yl)thio)acetohydrazide) was synthesized by adding hydrazine hydrate to compound (1) and refluxed for 3-4 h in propan-2-ol. Compound (3) characterized by ${ }^{1} H$-NMR peak, as a singlet due to $\left(\mathrm{NH}_{2}\right)$ at $\delta=2.01 \mathrm{ppm}$.

Synthesis of (5a-s) was carried out by adding aromatic or heterocyclic aldehyde to compound (3) in acetic acid. It is known, that this reaction proceeds by the mechanism of nucleophilic attachment of an amine to an aldehyde or ketone carbon. The attachment of the nucleophile to the carbonyl compound proceeds through the bipolar ion, as a result unstable heme amino alcohol is synthesized, which dehydrates to the final product. The signals of ${ }^{1} H$-NMR for (5a-s) are consented with the proposed structure.

Analyzing the LS/MS chromatogram in the MS spectrum there are molecular peaks with a value of 270 and $403(\mathrm{~m} / \mathrm{z})$, which corresponds to the calculated theoretical value of 2-((3-(thiophen-2ylmethyl)-1H-1,2,4-triazol-5-yl)thio)acetohydrazide (3) (left) and 3-nitrobenzylidene-2-((5-(thiophen2-ylmethyl)-4H-1,2,4-triazol-3-yl)thio)acetohydrazide (5j) (right) (Fig. 1)
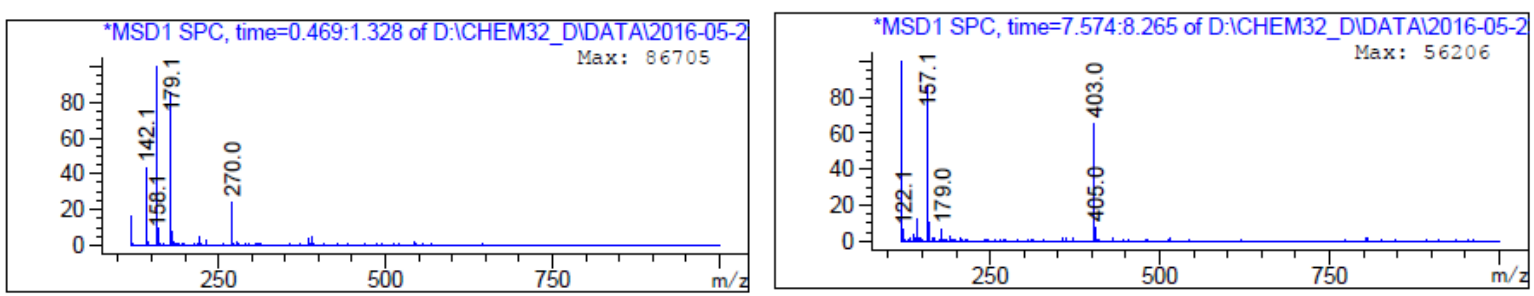

Fig. 1. Mass spectrum of 2-((3-(thiophen-2-ylmethyl)-1H-1,2,4-triazol-5-yl)thio)acetohydrazide (3) (left) and 3-nitrobenzylidene-2-((5-(thiophen-2-ylmethyl)-4H-1,2,4-triazol-3-yl)thio)acetohydrazide (5j) (right).

The elemental analysis (CHNS) was accomplished for synthesized compounds to confirm their basic chemical structures and revealed acceptable agreement with the calculated percentages.

The novel N'-substituted-2-((5-(thiophen-2-ylmethyl)-4H-1,2,4-triazol-3-yl)thio) acetohydrazides were synthesized and characterized.

\section{ACKNOWLEDGEMENT}

We're grateful to the Zaporizhzhia State Medical University for providing some facilities in carrying out the research. 


\section{REFERENCES}

1. Pattan, S., Gadhave, P., Tambe, V., Dengale, S., Thakur, D., Hiremath, S. V., \& Deotarse, P. (2012). Synthesis and evaluation of some novel 1, 2, 4-triazole derivatives for antmicrobial, antitubercular and anti-inflammatory activities. Indian Journal of Chemistry Section B 51(1):297301.

2. Shcherbyna, R. A., Panasenko, A. I., Knysh, E. G., \& Varinsky, B. A. (2014). Synthesis and physicochemical properties of 2-((4-R-3-(morpholinomethylene)-4H-1,2,4-triazol-5yl)thio)acetic acids. Aktual'nì Pitannâ Farmacevtičnoï ì Medičnoï Nauki ta Praktiki, 3 (16), 18 21.

3. Shcherbyna, R. A., Panasenko, A. I., Knysh, E. G., \& Varinsky, B. A. (2014). Synthesis and physicochemical properties of 2-((4-R-3-(morpholinomethylene)-4H-1,2,4-triazol-5yl)thio)acetic acids. Aktual'ni Pitannâ Farmacevtičnoï i Medičnoï Nauki ta Praktiki, 3 (16), 18 21.

4. Bushueva, I, Parchenko, V , Shcherbyna, R, Safonov, A, Kaplaushenko, A, Gutyj, B , Hariv, I . (2017). Tryfuzol - New original veterinary drug. Journal of Faculty of Pharmacy of Ankara University , 41 (1) , 0-0 . DOI: 10.1501/Eczfak_0000000594

5. Tkachenko, A., Zazharsky, V., Bilan, M., \& Kovaleva, L. (2010). Some peculiarities of bovis tuberculosis display in long not favourably farm. News of Dniproptrovsk State Agrarian and Economic University, (1), 100-103

6. Shcherbyna, R . (2019). Microwave-assisted synthesis of some new derivatives of 4-substituted3-(morpholinomethyl)-4h-1,2,4-triazole-5-thioles. Journal of Faculty of Pharmacy of Ankara University , 43 (3) , 220-229 . DOI: 10.33483/jfpau.533166.Shcherbyna, R. O. (2014). Analysis of pharmacological activity of derivatives of 1,2, 4-triazoles. Farmatsevtychnyi chasopys, 4, 145150.

7. Thakkar, S. S., Thakor, P., Doshi, H., \& Ray, A. (2017). 1,2,4-triazole and 1,3,4-oxadiazole analogues: synthesis, MO studies, in silico molecular docking studies, antimalarial as DHFR inhibitor and antimicrobial activities. Bioorganic \& Medicinal Chemistry. 25(15):4064-4075. doi: 10.1016/j.bmc.

8. Kumar, G. S., Prasad, Y. R., \& Chandrashekar, S. M. (2013). Synthesis and pharmacological evaluation of novel 4-isopropylthiazole-4-phenyl-1, 2, 4-triazole derivatives as potential antimicrobial and antitubercular agents. Medicinal Chemistry Research, 22(2), 938-948.

9. Patel, N. B., Khan, I. H., Pannecouque, C., \& De Clercq, E. (2013). Anti-HIV, antimycobacterial and antimicrobial studies of newly synthesized 1,2, 4-triazole clubbed benzothiazoles. Medicinal Chemistry Research, 22(3), 1320-1329.

10. Aksonova, I. I., Shcherbyna, R. O., Panasenko, O. I., Knysh, Y. H., \& Aksonov, I. V. (2014). The investigation of growth-stimulating activity of derivatives of 1,2,4-triazole on seeds of sunflower simple. Ukrainskyi biofarmatsevtychnyi zhurnal, 6, 78-82.

11. Rud, A. M., Kaplaushenko, A. G., Pruglo, Y. S., \& Frolova, Y. S. (2018). Establishment of diuretic activity indicators for (3-Thio-4-R-4-H-1,2,4-triazole-5-yl)(phenyl)methanols And Their Derivatives. Aktual'nì Pitannâ Farmacevtičnoï ì Medičnoï Nauki ta Praktiki, 2018(2), 215-219. 
12. Pilyugina K.S., Safonov A.A., Panasenko O.I., Knysh E.G., (2017). Synthesis, antimicrobial and antifungal activity of 2-((5-(thiophene-2-ylmethyl)-4N-1,2,4-triazoles-3-yl)thio)acetic acid esters. Materials \& Intern. Research Practice Conf. "Modern problems of pharmacotherapy and prescription of drugs". H., p.264.

13. Varynskyi, B., Knysh, Y., Parchenko, V., Panasenko, O., \& Kaplaushenko, A. (2015). The study of retention regularities for the potential drug substances of 1,2,4-triazol-3-ylthioacetic acids and their salts series by the method of HPLC/DAD-MS. Journal Of Organic And Pharmaceutical Chemistry, 13(4), 68-75.

14. Saidov, G. V., Sverdlova, O. V., \& Bakhshiev, N. G. (1995). Prakticheskoe rukovodstvo po molekuliârnoì spektroskopii: uchebnoe posobie. Izd-vo Sankt-Peterburgskogo universiteta

15. Kazitsena, L. and Kupletskaya, N. (1979). Primenenie UF-, IK-, YaMR - i MASS - spectrometrii $v$ orhanicheskoy khimii. 2nd ed. Moskow: University of Moscow, p.240. 